\title{
Severe hypoglycaemia and cognitive impairment in older patients with diabetes: the Fremantle Diabetes Study
}

\author{
D. G. Bruce • W. A. Davis • G. P. Casey • \\ R. M. Clarnette • S. G. A. Brown • I. G. Jacobs • \\ O. P. Almeida • T. M. E. Davis
}

Received: 4 April 2009 /Accepted: 4 June 2009 /Published online: 3 July 2009

(C) Springer-Verlag 2009

\begin{abstract}
Aims/hypothesis The aim was to investigate the relationship between severe hypoglycaemia and cognitive impairment in older patients with diabetes.

Methods A sample of 302 diabetic patients aged $\geq 70$ years was assessed for dementia or cognitive impairment without dementia in 2001-2002 and a subsample of non-demented patients $(n=205)$ was followed to assess cognitive decline. A history of severe hypoglycaemia was determined from self-reports, physician assessments and records of health service use for hypoglycaemia (HSH). Prospective HSH was determined up to 2006. Data analysis, including multiple logistic and Cox regression models, was used to determine whether: (1) there were cross-sectional associations between hypoglycaemia and cognitive status, (2) historical hypoglycaemia predicted cognitive decline, and (3) baseline cognitive status predicted subsequent HSH.
\end{abstract}

D. G. Bruce $(\varangle) \cdot$ W. A. Davis $\cdot$ G. P. Casey $\cdot$ T. M. E. Davis

School of Medicine and Pharmacology,

University of Western Australia, Fremantle Hospital,

PO Box 480, Fremantle, WA 6959, Australia

e-mail: dbruce@cyllene.uwa.edu.au

R. M. Clarnette

Department of Community and Geriatric Medicine,

Fremantle Hospital,

Fremantle, Australia

S. G. A. Brown • I. G. Jacobs

School of Primary, Aboriginal and Rural Health Care,

University of Western Australia,

Perth, WA, Australia

O. P. Almeida

School of Psychiatry and Neurosciences,

University of Western Australia and the Western Australian

Centre for Health and Ageing,

Perth, WA, Australia
Results There were significant cross-sectional associations between both cognitive impairment and dementia and hypoglycaemia. Independent risk factors for future $\mathrm{HSH}$ included dementia (hazard ratio 3.00, 95\% CI 1.06-8.48) and inability to self-manage medications (hazard ratio 4.17, 95\% CI 1.43-12.13). However, there were no significant associations between historical hypoglycaemia, incident HSH and cognitive decline.

Conclusions/interpretation Dementia is an important risk factor for hypoglycaemia requiring health service utilisation. We found no evidence that hypoglycaemia contributes to cognitive impairment in older patients with diabetes.

Keywords Cognitive decline - Cognitive impairment . Dementia $\cdot$ Diabetes $\cdot$ Severe hypoglycaemia

\author{
Abbreviations \\ CDR Clinical Dementia Rating \\ CVD Cerebrovascular disease \\ DSM Diagnostic and Statistical Manual \\ ED Emergency department \\ FDS Fremantle Diabetes Study \\ HSH Health service use for hypoglycaemia \\ PAD Peripheral arterial disease \\ WADLS Western Australia Data Linkage System
}

\section{Introduction}

Although there is little evidence that hypoglycaemic episodes complicating type 1 diabetes adversely affect brain function $[1,2]$, the situation may be different in older people with diabetes. This latter group has an increased risk of cognitive impairment [3], which may be associated with 
insulin therapy $[4,5]$. Since the association between diabetes and cognitive impairment appears confined to middle-aged or older individuals [6], the ageing brain could be particularly susceptible to neuroglycopenia. The interpretation of studies examining the relationship between blood glucose-lowering therapies, hypoglycaemia and cognitive function in type 2 diabetes is, however, complicated by factors other than age, such as duration of diabetes and of insulin treatment, comorbidities, including renal insufficiency, and polypharmacy [7-10]. In addition, ascertainment of hypoglycaemia in older patients can be underestimated because of less distinctive symptoms [11]. Cognitive impairment may itself lead to unreliable reporting of hypoglycaemia [12] and increase the risk of hypoglycaemia through medication errors and erratic meal patterns [9].

There are few detailed prospective studies of hypoglycaemia and cognition in older patients $[3,11]$. We have previously observed cross-sectional associations between self-reported severe hypoglycaemia and cognitive impairment in an elderly sample of patients with type 2 diabetes [13]. In the present study, we examined detailed data on the predictors of hypoglycaemia, including a requirement for medical/paramedical attention, to determine whether (1) a history of severe hypoglycaemia predicts subsequent cognitive decline, and (2) a diagnosis of cognitive impairment or dementia predicts future health service use for hypoglycaemia (HSH).

\section{Methods}

Patients

A consort diagram showing patient selection and recruitment to the present study is shown in Fig. 1. The Fremantle Diabetes Study (FDS) recruited 1,426 diabetic patients (63\%) from 2,258 identified in a postcode-defined urban Australian community between 1993 and 1996. Details of the FDS, including patient characteristics and methods used to diagnose diabetes, have been described previously [14]. In February 2001, there were 587 FDS survivors aged $\geq 70$ years, of whom $302(51.4 \%)$ agreed to undergo cognitive assessment. These latter patients comprised the present study sample, which was predominantly community-based with relatively few participants from residential care [13]. The 246 patients in this sample who were non-demented at the initial assessment and alive 18 months later were invited to a second cognitive assessment and $205(83.3 \%)$ attended [15]. The Human Research Ethics Committee of Fremantle Hospital approved the FDS and the present study, and all participants gave written informed consent.
Baseline assessment

Detailed sociodemographic, clinical and biochemical data were available at FDS entry for the present 302 patients and these data were updated on recruitment to the present study [14]. In addition, the FDS database was linked to the Western Australia Data Linkage System (WADLS) [16]. The WADLS records all hospitalisations and deaths in the state of Western Australia, and morbidity/mortality data from January 1993 until the end of June 2006 were extracted for the present sample.

Complications were identified using standard definitions $[14,17]$. Peripheral neuropathy was defined as a score $>2 / 8$ on the clinical portion of the Michigan Neuropathy Screening Instrument. Self-reported stroke/transient ischaemic attack amalgamated with prior hospitalisations defined cerebrovascular disease (CVD) status. Patients were classified with CHD if there was a self-reported history of or hospitalisation for myocardial infarction (MI), angina, revascularisation and/or definite MI on a Minnesota-coded electrocardiogram. Peripheral arterial disease (PAD) was defined by an ankle:brachial index $\leq 0.90$. Nephropathy was defined as a first-morning urinary albumin:creatinine ratio $\geq 3.0 \mathrm{mg} / \mathrm{mmol}$. The estimated glomerular filtration rate (eGFR) was calculated using the Modification of Diet in Renal Disease Study equation, with an eGFR $<60 \mathrm{ml} \mathrm{min}^{-1}$ $1.73 \mathrm{~m}^{-2}$ defining renal impairment [18]. Depression was assessed using the Even Briefer Assessment Scale for Depression [19]. Basic and instrumental activities of daily living (telephone use, transport, financial affairs, managing medications) were recorded. A BMI $<22 \mathrm{~kg} / \mathrm{m}^{2}$ defined participants at risk of under-nutrition [20].

Cognitive assessment

A two-step cognitive assessment was used as previously described [13, 15]. Briefly, all participants were screened using the Mini-Mental State Examination (MMSE), the Informant Questionnaire for Cognitive Decline in the Elderly (IQCODE), and a question on subjective memory. Those with a MMSE score $<28 / 30$ or with an IQCODE rating $\geq 3.31$, or who reported subjective memory loss, underwent a detailed cognitive assessment which was followed by a clinical review to establish the diagnosis of dementia according to the Diagnostic and Statistical Manual of Mental Disorders, Fourth Edition (DSM-IV) criteria. An additional random sample of 34 participants who screened negative completed the cognitive assessment and clinical review. They were all found to be cognitively normal.

Ratings of functional capacity were conducted by a trained researcher using the Clinical Dementia Rating (CDR) scale. A global CDR is derived by an algorithm 
Fig. 1 Flow diagram summarising sample recruitment and participation

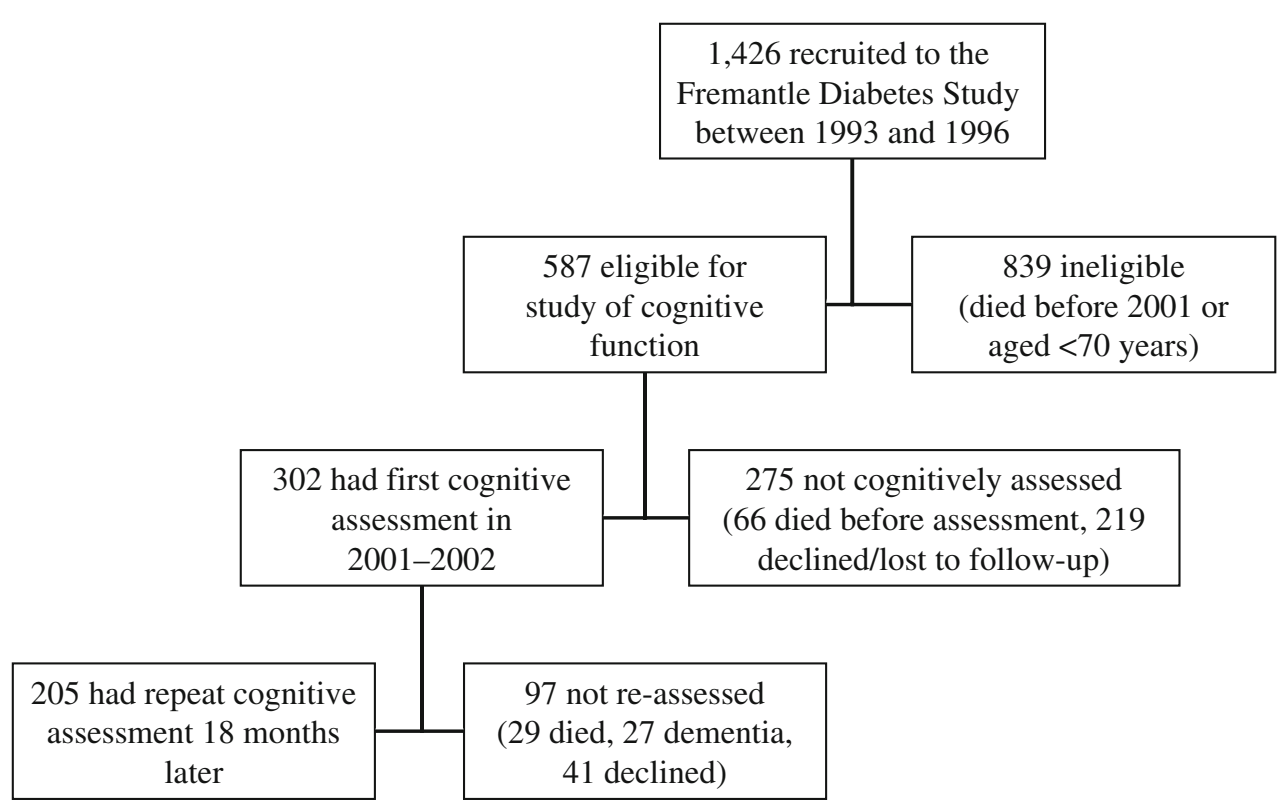

using a computer-based scoring program from ratings of six domains (memory, orientation, judgement, community affairs, home/hobbies, personal care) that are obtained from the patient and an informant [21]. The CDR includes an intermediate state between normal cognition and dementia $(\mathrm{CDR}=0.5)$, sometimes called early/questionable dementia, that was designated cognitive impairment without dementia in the present study because these participants did not have dementia according to DSM-IV. The screen-negative participants who underwent full assessment had normal cognition [13]. Consensus diagnoses were reached at review meetings of the investigators. We used the same approach described above to assess cognitive impairment at the 18-month clinical review, with 'cognitive decline' being defined as a change in classification from either normal cognition to dementia/cognitive impairment without dementia or from cognitive impairment without dementia to dementia [15].

\section{Ascertainment of hypoglycaemia}

Hypoglycaemic episodes were ascertained using three distinct approaches.

Self-report A trained research nurse asked participants 'Have you ever had to go to hospital because of a hypoglycaemic attack?' and 'Have you ever had a serious hypoglycaemic attack that made you go unconscious?' A positive response to either question identified self-reported severe hypoglycaemia and led to follow-up questions on the frequency and approximate dates to facilitate confirmatory review of hospital records.
Doctor verification All participants seen by study physicians for cognitive assessment were assessed using a checklist that included questions on history, frequency, symptoms [10] and severity of hypoglycaemia, and whether there was confirmation with blood glucose measurement below the normoglycaemic range $(<3.5 \mathrm{mmol} / \mathrm{l})$ [9]. The physician rated the accuracy of reports as proven, probable or uncertain. In the present study, proven and probable episodes were classified as doctor-verified histories of any hypoglycaemia, severe hypoglycaemia (episodes that required second-party assistance because of an inability to self-treat) and neuroglycopenia (episodes with appropriate symptoms).

HSH This was defined as an event requiring ambulance and/or emergency department (ED) attendance and/or hospitalisation for hypoglycaemia as the primary diagnosis. All HSH episodes in the WADLS between January 1999 and the end of June 2006 inclusive were identified. A separate database for Fremantle Hospital ED attendances was available from January 1999 to December 2003 and for all metropolitan EDs from July 2001 to June 2006. Hypoglycaemia was identified as a problem code of 'hypoglycaemia' and/or treatment with glucagon from the ambulance database. An ED presentation was defined according to the International Classification of Diseases (ICD)-10 (http://www.who.int/classifications/icd/en/) diagnosis codes for hypoglycaemia and/or description of the diagnosis and/or presenting symptoms. The case notes of patients hospitalised with ICD-9 or -10 codes relating to hypoglycaemia were reviewed where possible to validate the coding. 
Data analysis

The computer packages SPSS for Windows (version 15.0; SPSS, Chicago, IL, USA) and Intercooled Stat (version 10.0; Stata Corp., College Station, TX, USA) were used for statistical analyses. Baseline characteristics were analysed (1) to establish whether measures of prior hypoglycaemia were associated with initial cognitive impairment and/or subsequent cognitive decline, and then (2) to identify risk factors for HSH occurring after initial assessment. Data are presented as proportions, mean $\pm \mathrm{SD}$, geometric mean (SD range) or, in the case of variables which did not conform to a normal or lognormal distribution, median and interquartile range (IQR). Two-sample comparisons of proportions were by Fisher's exact test, for normally distributed variables by Student's $t$ test, and for non-normally distributed variables by the Mann-Whitney $U$ test. Comparisons of more than two groups were by $\chi^{2}$ or Fisher's exact test, ANOVA or Kruskal-Wallis test.

For multiple logistic regression analysis we used forward conditional modelling $(p<0.05$ for entry and $>0.10$ for removal), and clinically plausible variables with $p<0.20$ in the respective univariate analyses were entered into relevant models. Kaplan-Meier plots of $\mathrm{HSH}-$ free survival by cognitive status were compared by the logrank test. Cox proportional hazards modelling with forward conditional variable entry $(p<0.05)$ and removal $(p>0.10)$ was used to determine independent predictors of first HSH episode after study entry. In the model, there was no significant interaction between $\log _{e}$ (time) and any independent determinant, and their respective $\log _{e}$ ( $-\log _{e}[$ survival] $)$ curves were parallel, indicating that the proportional hazards assumption was valid. Predictors of frequency of $\mathrm{HSH}$ were assessed using a negative binomial regression model. To allow for the skewed distribution of duration of follow-up, an offset variable of the natural $\log$ arithm $\left(\log _{e}\right)$ of the total follow-up time for each patient was used.

\section{Results}

Sample characteristics

The present sample had a mean $( \pm \mathrm{SD})$ age of $76.0 \pm$ 4.6 years and all except nine patients $(3.0 \%)$ lived within the community. Almost all $(99.0 \%)$ had type 2 diabetes (27.5\% were on insulin and $45.0 \%$ were on sulfonylurea drugs) and $46.4 \%$ had an $\mathrm{HbA}_{1 \mathrm{c}} \leq 7.0 \%$. Dementia was present in $9.3 \%$ and cognitive impairment without dementia in $19.9 \%$.
Prior hypoglycaemia and cognition at study entry

A history of severe hypoglycaemia was self-reported by $7.2 \%$ of the sample. This first occurred a median 1.0 (IQR 1.0-6.5) years before cognitive assessment. In the 200 patients seen by physicians (cognitive screen-positive plus 34 screen-negative patients), doctor-verified episodes of any hypoglycaemia had occurred in $39.5 \%$, with severe hypoglycaemia in $7.0 \%$ and neuroglycopenia in $8.0 \%$. HSH had occurred in $3.6 \%$, a median of $1.7(0.3-4.0)$ years prior to cognitive assessment. The prevalence of hypoglycaemia in each category is summarised by cognitive status in Table 1. Severe hypoglycaemia was at least three times more frequent in patients with cognitive impairment or dementia than in those with normal cognition regardless of the method of ascertainment, older adults with dementia having substantially increased odds of a doctor-verified or HSH-documented episode.

Cross-sectional associations between hypoglycaemic events and cognitive categories (normal cognition vs each of dementia, cognitive impairment without dementia, and both cognitive impairment categories combined) were examined using multiple logistic regression analysis. Each model was adjusted for previously established risk factors (age, sex, education and CVD for cognitive impairment without dementia; PAD and duration of diabetes for dementia; CVD, PAD and duration of diabetes for all cognitive impairment [13]). There were no significant independent associations between dementia and any measure of hypoglycaemia $(p>0.10)$. However, cognitive impairment without dementia was independently associated with self-reported severe hypoglycaemia (odds ratio $[95 \% \mathrm{CI}] 2.96(1.05-8.33), p=0.040)$, doctorverified neuroglycopenia (5.10 [1.46-17.87], $p=0.011)$ and HSH (9.65 [1.65-56.60], $p=0.012)$. The combination of both cognitive impairment categories was independently associated with doctor-verified severe hypoglycaemia (4.83 [1.29-18.13], $p=0.020)$, doctor-verified neuroglycopenia (5.04 [1.53-16.57], $p=0.008)$ and HSH (6.44 [1.22-34.14], $p=0.029)$.

Prior hypoglycaemia, incident $\mathrm{HSH}$ and cognitive decline

Of 205 non-demented participants who underwent followup a median 1.6 (1.4-1.8) years later, 33 (16.1\%) experienced cognitive decline during this period with four new cases of dementia and 29 new cases of cognitive impairment without dementia (Table 2). The patients who experienced cognitive decline were significantly older than those who did not but there was no significant difference in prior hypoglycaemia between the two groups. One of the five non-demented patients with incident $\mathrm{HSH}(20.0 \%)$ suffered cognitive decline between assessments, while 32 
Table 1 Baseline characteristics and categories of hypoglycaemia by cognitive status in 302 older participants with diabetes

Data are mean \pm SD or median (interquartile range)

Patients with cognitive impairment were non-demented

${ }^{\mathrm{a}} n=200$ (133 normal, 43 cognitively impaired, 24 with dementia)

$* p<0.05, * * p<0.01$, $* * * p<0.001$ compared with normal cognition group

\begin{tabular}{|c|c|c|c|c|}
\hline Characteristic & $\begin{array}{l}\text { Normal } \\
(n=214)\end{array}$ & $\begin{array}{l}\text { Cognitive impairment } \\
(n=60)\end{array}$ & $\begin{array}{l}\text { Dementia } \\
(n=28)\end{array}$ & $p$ value \\
\hline Age (years) & $75.4 \pm 4.4$ & $76.5 \pm 4.5$ & $79.6 \pm 5.0^{* *}$ & $<0.001$ \\
\hline Sex (\% male $)$ & 44.4 & $61.7 *$ & 50.0 & 0.060 \\
\hline Type 2 diabetes $(\%)$ & 99.1 & 98.3 & 100 & 0.75 \\
\hline Diabetes duration (years) & $10.8(8.3-15.4)$ & $12.1(8.6-18.1)$ & $12.5(9.6-21.4)^{*}$ & 0.058 \\
\hline Diet treatment $(\%)$ & 18.2 & 11.7 & 17.9 & 0.48 \\
\hline Oral hypoglycaemic agents (\%) & 57.5 & 53.3 & 46.4 & 0.50 \\
\hline Insulin \pm oral agents $(\%)$ & 24.3 & 35.0 & 35.7 & 0.15 \\
\hline Fasting glucose (mmol/l) & $8.0(6.5-9.7)$ & $8.0(6.3-9.9)$ & $8.2(6.6-9.8)$ & 0.96 \\
\hline $\mathrm{HbA}_{1 \mathrm{c}}(\%)$ & $7.1(6.5-8.0)$ & $7.2(6.6-8.3)$ & $7.3(6.7-8.3)$ & 0.49 \\
\hline BMI $\left(\mathrm{kg} / \mathrm{m}^{2}\right)$ & $28.6 \pm 4.7$ & $29.4 \pm 4.2$ & $26.6 \pm 4.7$ & 0.050 \\
\hline \multicolumn{5}{|l|}{ Prior hypoglycaemia } \\
\hline Self-reported severe $(\%)$ & 4.3 & $14.0^{*}$ & $16.0^{*}$ & 0.009 \\
\hline \multicolumn{5}{|l|}{ Doctor-verified $^{\mathrm{a}}$} \\
\hline Any $(\%)$ & 37.6 & 51.2 & 29.2 & 0.156 \\
\hline Severe $(\%)$ & 3.0 & $11.6^{*}$ & $20.8^{* *}$ & 0.003 \\
\hline Neuroglycopenia (\%) & 3.8 & $16.3^{*}$ & $16.7^{*}$ & 0.008 \\
\hline Ambulance/ED/hospital (\%) & 0.9 & 6.7 & $17.9 * * *$ & $<0.001$ \\
\hline
\end{tabular}

of the $200(16.0 \%)$ without HSH exhibited cognitive decline $(p=0.59)$.

\section{Determinants of HSH during follow-up}

A total of 27 of the 302 patients $(8.9 \%)$ had 40 episodes of HSH between initial cognitive assessment and the end of June 2006 during 1,128 patient-years of follow-up (mean $3.7 \pm 1.3$ years) to first occurrence of $\mathrm{HSH}$ or death or 30 June 2006, whichever came first. The crude incidence of HSH was 3.5 episodes/100 patient-years. Key baseline clinical and cognitive variables in those who did and did not experience HSH are shown in Table 3. There were significant differences between the groups in diabetes duration, walking ability, independence with finances and medications, dementia, insulin treatment, BMI $<22 \mathrm{~kg} / \mathrm{m}^{2}$ and history of severe hypoglycaemia. Non-significant variables included marital status, basic activities of daily living, depression, fasting glucose, serum cholesterol, CVD, CHD, PAD and nephropathy.

Demented patients had a significantly higher risk of HSH during follow-up (Fig. 2; $p<0.001$, logrank test). In Cox proportional hazards modelling with time to first $\mathrm{HSH}$ episode as the dependent variable, the significant independent baseline determinants were dementia (hazard ratio [95\% CI] 3.02 [1.07-8.53], $p=0.037)$, insulin therapy $(2.77$
Table 2 Baseline characteristics, including prior hypoglycaemia, of 205 non-demented participants categorised by change in cognitive status over a median of 1.6 years

Data are mean $\pm \mathrm{SD}$ or median (interquartile range)

${ }^{\mathrm{a}} n=161$ with doctor interview, 134 with no cognitive decline, 27 with cognitive decline

\begin{tabular}{llll}
\hline Characteristic & $\begin{array}{l}\text { No cognitive decline } \\
(n=172)\end{array}$ & $\begin{array}{l}\text { Cognitive decline } \\
(n=33)\end{array}$ & $p$ value \\
\hline Age (years) & $74.7 \pm 4.0$ & $76.3 \pm 4.4$ & 0.037 \\
Sex (\% male) & 50.0 & 39.4 & 0.34 \\
Duration diabetes (years) & $11.1(8.3-16.1)$ & $9.6(7.9-15.1)$ & 0.30 \\
Diet treatment (\%) & 15.7 & 24.2 & 0.31 \\
Oral hypoglycaemic agents (\%) & 55.2 & 63.6 & 0.45 \\
Insulin \pm oral hypoglycaemic agents (\%) & 29.1 & 12.1 & 0.052 \\
HbA $_{1 \mathrm{c}}(\%)$ & $7.0(6.5-7.9)$ & $7.5(6.3-8.2)$ & 0.64 \\
Prior hypoglycaemia & & & \\
Self-reported severe (\%) & 6.6 & 9.1 & 0.71 \\
Doctor-verified & & & \\
$\quad$ Any (\%) & 44.0 & 25.9 & 0.09 \\
$\quad$ Severe (\%) & 6.0 & 0.0 & 0.35 \\
$\quad$ Neuroglycopenia (\%) & 6.0 & 7.4 & 0.68 \\
$\quad$ Ambulance/ED/hospital (\%) & 2.3 & 0.0 & 1.00 \\
\hline
\end{tabular}


Table 3 Baseline characteristics of 302 diabetic participants who were followed for subsequent hypoglycaemia requiring ambulance, emergency department or hospital services
Data are mean \pm SD or median and (interquartile range)

${ }^{\mathrm{a}} n=200$ participants

\begin{tabular}{|c|c|c|c|}
\hline Characteristic & $\begin{array}{l}\text { No severe } \\
\text { hypoglycaemia } \\
(n=275)\end{array}$ & $\begin{array}{l}\text { Severe } \\
\text { hypoglycaemia } \\
(n=27)\end{array}$ & $p$ value \\
\hline Age (years) & $75.9 \pm 4.6$ & $76.9 \pm 4.6$ & 0.29 \\
\hline Sex $(\%$ male $)$ & 48.0 & 51.9 & 0.84 \\
\hline Duration of diabetes (years) & $10.9(8.3-15.7)$ & $15.9(9.8-20.8)$ & 0.007 \\
\hline Uses walking aid (\%) & 23.8 & 48.1 & 0.010 \\
\hline Independent with finances $(\%)$ & 85.5 & 63.0 & 0.006 \\
\hline Independent with medications (\%) & 89.3 & 66.7 & 0.003 \\
\hline Dementia $(\%)$ & 6.9 & 33.3 & $<0.001$ \\
\hline Cognitive impairment without dementia & 20.4 & 14.8 & 0.62 \\
\hline Treatment with sulfonylureas (\%) & 45.0 & 45.8 & 0.94 \\
\hline Insulin \pm oral agents & 24.7 & 55.6 & 0.001 \\
\hline $\mathrm{HbA}_{1 \mathrm{c}}(\%)$ & $7.1(6.5-8.0)$ & $7.7(6.8-8.4)$ & 0.07 \\
\hline BMI $<22 \quad \mathrm{~kg} / \mathrm{m}^{2}(\%)$ & 5.3 & 16.7 & 0.053 \\
\hline Lipid-lowering drugs (\%) & 55.4 & 33.3 & 0.042 \\
\hline Estimated GFR $<60 \mathrm{ml} \min ^{-1} 1.73 \mathrm{~m}^{-2}(\%)$ & 37.5 & 51.9 & 0.15 \\
\hline Prior self-reported severe hypoglycaemia (\%) & 6.1 & 18.5 & 0.034 \\
\hline Prior doctor-verified severe hypoglycaemia $(\%)^{\mathrm{a}}$ & 3.4 & 40.0 & $<0.001$ \\
\hline Prior doctor-verified neuroglycopenia $(\%)^{\mathrm{a}}$ & 4.5 & 38.1 & $<0.001$ \\
\hline Prior hospital services for hypoglycaemia (\%) & 2.2 & 18.5 & 0.037 \\
\hline
\end{tabular}

[1.18-6.46], $p=0.019)$, low BMI (5.94 [1.85-19.06], $p=$ $0.003)$, inability to self-manage medications (4.19 [1.4312.25], $p=0.009$ ) and a history of self-reported severe hypoglycaemia (3.51 [1.15-10.76], $p=0.028)$.

Patients who experienced HSH had an average of $1.5 \pm$ 0.9 (range 1-4) events during follow-up. In a negative binomial regression model, significant predictors of the frequency of HSH were dementia (relative risk [95\% CI] 20.26 [6.00-68.44], $p<0.001)$, insulin therapy (14.60 [3.49-61.12], $p<0.001)$ and renal impairment (4.70 [1.0221.70], $p=0.048)$.

\section{Discussion}

We found that severe hypoglycaemia was common in our sample of largely community-based older Australians with diabetes. Based on simple self-report and doctor interpretation of events, one in 14 patients had experienced an episode within a few years of assessment and nearly half of these patients needed access to health services as a result. Prior hypoglycaemia and incident severe hypoglycaemia were not associated with cognitive decline in patients with normal cognition at baseline. However, baseline dementia was a strong independent predictor of HSH over the subsequent 5 years. These data suggest that severe hypoglycaemia does not cause or contribute to cognitive impairment and dementia in diabetes but that older diabetic patients with dementia are at substantially increased risk of HSH.
The lack of an association between prior severe hypoglycaemia (self-reported, doctor-verified or HSH) and subsequent cognitive decline in our subgroup of 205 patients with normal cognition at baseline suggests that, as in type 1 diabetes $[1,2]$, in older people with diabetes hypoglycaemia does not have persistent adverse neurological effects. Nevertheless, it remains possible that patients with established cognitive impairment may be particularly susceptible to the irreversible effects of severe hypogly-

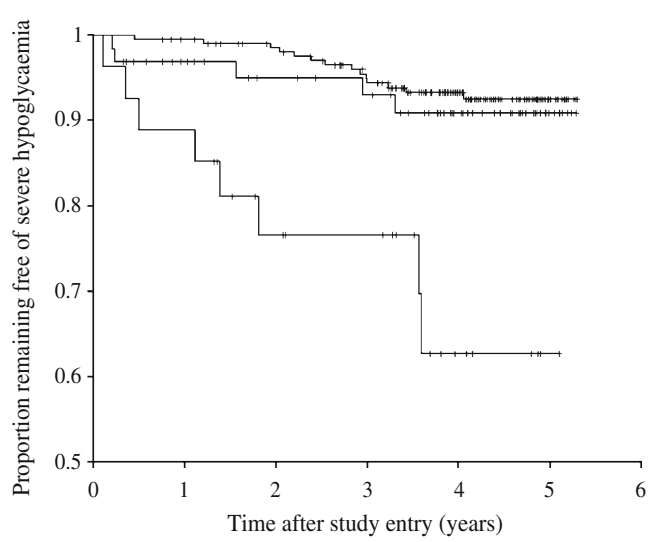

Fig. 2 Survival probability curves derived from Kaplan-Meier analysis of proportions of patients remaining free of severe hypoglycaemia defined by cognitive status at study entry. The upper curve is that for patients with normal cognition at baseline, the middle line for those with cognitive impairment and the lower curve that for demented patients. In each case, censored data points are indicated by crosses 
caemia, an association that we were unable to verify in the present study. An examination of this issue would have to take into consideration factors such as the relative severity of individual episodes. The well recognised brain damage associated with repeated severe hypoglycaemia [22] may be more likely where symptom recognition and the ability to self-correct or call for assistance is impaired, such as in patients with cognitive impairment or dementia.

There is evidence that frail older patients are prone to severe hypoglycaemia $[7,8,23]$. Our data indicate that dementia is likely to be an additional major independent contributor to this excess risk with potentially adverse consequences for the individual and the community. Ambulance and hospital service use are expensive consequences of a preventable condition [23]. In addition, patients with dementia frequently suffer further cognitive and functional decline during hospitalisation regardless of the cause of admission [24]. The present data add weight to the need for cognitive screening of older patients with diabetes, especially since dementia is under-diagnosed by healthcare professionals [25]. Furthermore, our observation that dementia was strongly associated with HSH frequency suggests that measures to prevent recurrent episodes are inadequate. The involvement of family caregivers and formal support services would seem vital in demented patients in response to an episode of HSH.

We found additional novel risk factors for future HSH. A BMI $<22 \mathrm{~kg} / \mathrm{m}^{2}$ has been used to screen for undernutrition in older patients and is associated with several chronic conditions, including dementia [26]. Patients in this category in the present sample were six times more likely to access health services for hypoglycaemia than those with greater BMI. This observation might be explained by insufficient energy intake for the prescribed blood glucose-lowering treatment and/or erratic meal patterns [9], but such detailed dietary data were not collected in the present study. Insulin treatment, a well recognised risk factor for severe hypoglycaemia in the elderly [8-10], increased the risk of future HSH three-fold in the present study and was also a strong risk factor for recurrent $\mathrm{HSH}$. However, we also identified difficulties with selfmanagement of diabetes medications as a risk factor. These difficulties are a feature of dementia [27] but have not previously been associated with hypoglycaemia. They could reflect the severity of dementia or specific dementia-associated functional impairments.

The strengths of the present study are the use of all available data (subjective report, doctor-verification of episodes and documented health service utilisation) to ascertain severe hypoglycaemia, the representative community-based nature of the patients and the detailed data collection, which included all recognised risk factors. In addition, our crude incidence of HSH was consistent with those in other studies which used comparable methods of ascertainment [23, 28]. However, because the sample size was not large and the follow-up period was relatively short, the study may have had limited power to investigate the association between incident hypoglycaemia and cognitive decline. We used a categorical classification of cognitive change (adopted for theoretical and practical reasons) that precluded the detection of subtle global change or decline in specific domains. In addition, the use of self-reported hypoglycaemia, especially in patients with cognitive impairment, has recognised limitations [12], while even the most objective index of severe hypoglycaemia, HSH, can underestimate its frequency [10]. While our data argue against hypoglycaemia having a major impact on cognition, further study is required to exclude a more subtle association.

In a recent study using a large health database of insured US patients, a history of severe hypoglycaemia assessed from hospital discharge and emergency department data was associated with a greater risk of dementia in those with type 2 diabetes [29]. The diagnosis of dementia was based on inpatient and outpatient diagnosis codes rather than formal cognitive assessment, and no serial assessments of cognitive function were performed. Because the authors were uncertain of the temporality of the association and could not exclude reverse causality, their findings do not contradict those of the present study.

We conclude that dementia is an important risk factor for hypoglycaemia complicating diabetes in older patients. Clinical management in this group should include cognitive screening to identify patients that could benefit from strategies (such as supervised treatment and increased blood glucose monitoring) that reduce the risk of severe hypoglycaemia. While we found no evidence that hypoglycaemia adversely affects cognition, further detailed longitudinal studies are warranted.

Acknowledgements The study was carried out with support from an unrestricted educational grant from Sanofi-Aventis, the National Health and Medical Research Council of Australia (Project Grant Number 139139), the Diabetes Australia Research Trust and the Medical Research Foundation of Fremantle Hospital. The Fremantle Diabetes Study was funded by the Raine Foundation, University of Western Australia. T.M.E. Davis is supported by a National Health and Medical Research Council of Australia Practitioner Fellowship.

Duality of interest The authors declare that there is no duality of interest associated with this manuscript.

\section{References}

1. Jacobson AM, Musen G, Ryan CM et al (2007) Long-term effect of diabetes and its treatment on cognitive function. N Engl J Med 356:1842-1852 
2. Strudwick SK, Carne C, Gardiner J, Foster JK, Davis EA, Jones TW (2005) Cognitive functioning in children with early onset type 1 diabetes and severe hypoglycemia. J Pediatr 147:680-685

3. Cukierman T, Gerstein HC, Williamson JD (2005) Cognitive decline and dementia in diabetes-systematic overview of prospective observational studies. Diabetologia 48:2460-2469

4. MacKnight C, Rockwood K, Awalt E, McDowell I (2002) Diabetes mellitus and the risk of dementia, Alzheimer's disease and vascular cognitive impairment in the Canadian Study of Health and Aging. Dement Geriatr Cogn Disord 14:77-83

5. Ott A, Stolk RP, van Harskamp F, Pols HA, Hofman A, Breteler MM (1999) Diabetes mellitus and the risk of dementia: the Rotterdam Study. Neurology 53:1937-1942

6. Ryan CM, Geckle M (2000) Why is learning and memory dysfunction in type 2 diabetes limited to older adults? Diabetes Metab Res Rev 16:308-315

7. Seltzer HS (1972) Drug-induced hypoglycemia. A review based on 473 cases. Diabetes 21:955-966

8. Shorr RI, Ray WA, Daugherty JR, Griffin MR (1997) Incidence and risk factors for serious hypoglycemia in older persons using insulin or sulfonylureas. Arch Intern Med 157:1681-1686

9. Amiel SA, Dixon T, Mann R, Jameson K (2008) Hypoglycaemia in type 2 diabetes. Diabet Med 25:245-254

10. Hepburn DA, MacLeod KM, Pell AC, Scougal IJ, Frier BM (1993) Frequency and symptoms of hypoglycaemia experienced by patients with type 2 diabetes treated with insulin. Diabet Med 10:231-237

11. Jaap AJ, Jones GC, McCrimmon RJ, Deary IJ, Frier BM (1998) Perceived symptoms of hypoglycaemia in elderly type 2 diabetic patients treated with insulin. Diabet Med 15:398-401

12. Heller S, Chapman J, McCloud J, Ward J (1995) Unreliability of reports of hypoglycaemia by diabetic patients. BMJ 310:440

13. Bruce DG, Davis WA, Casey GP et al (2008) Predictors of cognitive impairment and dementia in older people with diabetes. Diabetologia 51:241-248

14. Davis TM, Zimmet P, Davis WA, Bruce DG, Fida S, Mackay IR (2000) Autoantibodies to glutamic acid decarboxylase in diabetic patients from a multi-ethnic Australian community: the Fremantle Diabetes Study. Diabet Med 17:667-674

15. Bruce DG, Davis WA, Casey GP et al (2008) Predictors of cognitive decline in older individuals with diabetes. Diabetes Care 31:2103-2107
16. Holman CD, Bass AJ, Rouse IL, Hobbs MS (1999) Populationbased linkage of health records in Western Australia: development of a health services research linked database. Aust N Z J Public Health 23:453-459

17. Norman PE, Davis WA, Bruce DG, Davis TM (2006) Peripheral arterial disease and risk of cardiac death in type 2 diabetes: the Fremantle Diabetes Study. Diabetes Care 29:575-580

18. Levey AS, Bosch JP, Lewis JB, Greene T, Rogers N, Roth D (1999) A more accurate method to estimate glomerular filtration rate from serum creatinine: a new prediction equation. Modification of Diet in Renal Disease Study Group. Ann Intern Med 130:461-470

19. Allen N, Ames D, Ashby D, Bennetts K, Tuckwell V, West C (1994) A brief sensitive screening instrument for depression in late life. Age Ageing 23:213-219

20. Tayback M, Kumanyika S, Chee E (1990) Body weight as a risk factor in the elderly. Arch Intern Med 150:1065-1072

21. Morris JC (1993) The Clinical Dementia Rating (CDR): current version and scoring rules. Neurology 43:2412-2414

22. Warren RE, Frier BM (2005) Hypoglycaemia and cognitive function. Diabetes Obes Metab 7:493-503

23. Leese GP, Wang J, Broomhall J et al (2003) Frequency of severe hypoglycemia requiring emergency treatment in type 1 and type 2 diabetes: a population-based study of health service resource use. Diabetes Care 26:1176-1180

24. Fick DM, Agostini JV, Inouye SK (2002) Delirium superimposed on dementia: a systematic review. J Am Geriatr Soc 50:17231732

25. Knopman DS (1998) The initial recognition and diagnosis of dementia. Am J Med 104:2S-12S discussion 39S-42S

26. Gillette-Guyonnet S, Nourhashemi F, Andrieu S et al (2000) Weight loss in Alzheimer disease. Am J Clin Nutr 71:637S-642S

27. Sinclair AJ, Girling AJ, Bayer AJ (2000) Cognitive dysfunction in older subjects with diabetes mellitus: impact on diabetes selfmanagement and use of care services. All Wales Research into Elderly (AWARE) Study. Diabetes Res Clin Pract 50:203-212

28. Holstein A, Plaschke A, Egberts EH (2003) Clinical characterisation of severe hypoglycaemia - a prospective population-based study. Exp Clin Endocrinol Diabetes 111:364-369

29. Whitmer RA, Karter AJ, Yaffe K, Quesenberry CP Jr, Selby JV (2009) Hypoglycemic episodes and risk of dementia in older patients with type 2 diabetes mellitus. JAMA 301:1565-1572 